\title{
Developing standards for the development of glaucoma virtual clinics using a modified Delphi approach
}

Aachal Kotecha $\mathrm{PhD} 1$

Simon Longstaff FRCOphth

Augusto Azuaro-Blanco FRCOphth

James Kirwan FRCOphth

James Morgan FRCOphth

Fiona Spencer FRCOphth

Paul Foster FRCOphth $-3^{\text {rd }}$ or senior

1. NIHR Biomedical Research Centre (BRC) at Moorfields Eye Hospital and UCL Institute of Ophthalmology

2. Belfast

3 Portsmouth

4 Royal Hallamshire Hospital, Sheffield

5 Cardiff

6 Manchester

Correspondence to:

Aachal Kotecha, NIHR Senior Research Associate, UCL Institute of Ophthalmology, 11-43 Bath Street, London, EC1V 9EL

Tel: +4476084015

Email: aachal.kotecha@ucl.ac.uk/ aachalkotecha@gmail.com

Word count:

Keywords:

Conflict of interest: The authors declare no conflict of interest 


\begin{abstract}
Purpose: To develop a set of standards framework for the development and implementation of virtual clinics for glaucoma monitoring in the United Kingdom (UK) using a modified Delphi methodology

Methods: A modified Delphi technique was used that involved sampling members of the United Kingdom Glaucoma and Eire Society (UKEGS). The first round involved scoring the strength of agreement to a series of standards statements using a 9-point Likert scale. The revised standards were subjected to a second round of scoring and free-text comment. The final standards were discussed and agreed by an expert panel consisting of 7 glaucoma subspecialists from across the country. A version of the standards was sent to external stakeholders for a 3 month consultation.
\end{abstract}

Results: There was a 44\% response rate of UKEGS members to round 1 and round 2, consisting largely of consultant ophthalmologists with a specialist interest in glaucoma. The final version of the standards document was validated by stakeholder consultation and contains 4 sections pertaining to the patient groups, testing methods, staffing requirements and governance structure of NHS secondary care glaucoma virtual clinic models.

Conclusions: Using a modified Delphi approach has resulted in consensus agreement for the standards required for the development of virtual clinics for monitoring glaucoma in the UK. It is anticipated that this document will be used as a guide for those setting up this model of service delivery in their department. 


\section{Background}

Chronic eye disease management is a particular challenge facing the United Kingdom (UK) National Health Service. The UK population is increasing, and predictions estimate that the UK population will have reached approximately 72 million by 2031 . Furthermore, as with most Western countries, the UK is an increasingly ageing population. [1] The increasing prevalence of glaucoma in the elderly population [2] and advances in diagnostic technologies used by primary care providers[3] means that the number of new glaucoma suspect referrals to secondary NHS care are increasing; $[4,5]$ however, the chronicity of these conditions means that patients often require lifelong NHS eye service care. As such most hospital eye departments have significant capacity problems with ophthalmology out-patient services. [6, 7] Most recognise that there is a need to redesign the relevant patient pathways in order to improve current capacity, reduce overdue review intervals and enhance patient experience of ophthalmology services. [8]

One solution is to adopt a 'virtual clinic' for managing stable patients.

A virtual clinic is one in which the face-to-face clinician consultation is removed. In a synchronous virtual clinic, the patient and clinician interact in real time, for example, via a webcam. In the asynchronous virtual clinic, the interaction occurs at different times. In a glaucoma asynchronous virtual clinic, technicians gather quantifiable data for clinicians to make value decisions. In this type of model, clinicians can review considerably more patient data than in traditional clinics. $[9,10]$

With little or no increased capital and recurrent costs, there is emerging evidence of the increasing popularity of virtual clinics within secondary care both for glaucoma and, increasingly, medical retina specialties to help deal with increased patient numbers. [1014] Technician-led virtual clinics may be placed at locations independent of the reviewing clinician so enhancing a Trust's ability to deliver CCG ideals of community based care.

There are no clear guidelines for the development of these services, and whilst there is some evidence suggesting that this model is equivalent to standard face-to-face care, 
[12] it is still a relatively new model of service delivery. Thus, as more Trusts start to implement these clinics to cope with their capacity issues, we felt it was necessary for experts in the field to define the standards expected of these clinics so that the quality of patient care within the NHS setting is maintained. [15]

The Delphi process is a technique widely adopted in social sciences and health research that seeks to obtain the agreement of experts in order to transform expert opinion into expert consensus.[16] We adopted a modified Delphi approach to define the standards required for the development and implementation of virtual clinics in glaucoma care. 


\section{Methods}

A convenience sample of 7 glaucoma subspecialists (6 consultant ophthalmologists and 1 glaucoma specialist optometrist) from across England, Northern Ireland and Wales formed the Delphi expert panel. For the first stage of the exercise, the panel were invited to comment upon a series of standards statements that had be prior drafted by the project steering group, which pertained to the patient groups, staffing requirements, test requirements and governance structure suitable or necessary for virtual glaucoma monitoring clinics. Once standards had been agreed by the panel, the project moved to the next stage which involved a 2 round survey of glaucoma clinicians.

\section{Round 1:}

A questionnaire was developed utilizing a web-based survey tool (SurveyMonkey® available at www.surveymonkey.com). The questionnaire consisted of 9 questions with a total of 25 statements that required scoring using a 9-point Likert scale indicating the strength of agreement with the statement, ranging from $9=$ 'strongly agree' to $1=$ 'strongly disagree'. The questionnaire was circulated to all members of the United Kingdom and Eire Glaucoma Society (UKEGS) who had provided their email address to UKEGS for the purposes of being involved in surveys. Six of the questions contained free text boxes for respondents to add any comments. All responses were anonymous.

The Delphi methodology does not specify at what point consensus is deemed to be achieved, thus, as per Myint et al, [17] we chose to exclude statements that scored $\leq 5$ from the standards. Free-text comments from round 1were also considered when revising the standards statements.

\section{Round 2:}

Following modifications resulting from the responses of round 1, the questionnaire was recirculated to UKEGS members as before. As in round 1, statements that scored $\leq 5$ were excluded from the final statements document.

Aversion of the statements document generated following round 2 was circulated to relevant stakeholders that included the Royal College of Ophthalmologists, the College of Optometrists, the Royal College of Nursing Ophthalmic Forum, The British and Irish 
Orthoptics Society and UK members of the World Association of Eye Hospitals.

Stakeholders were given a 3 month window to respond to the document.

The expert panel met once more to review stakeholder comments, and a final version of the standards document was developed.

\section{Results:}

Round 1

The survey was electronically circulated to 196 members of UKEGS for a 6 week period in June 2015, with 2 electronic reminders over this period. Eight-six members responded to the survey, with few undeliverable emails (personal communication, $\mathrm{T}$ Fabre, UKEGS Marketing Support \& Events Officer, 2 July 2015), which amounts to an approximate UKEGS member response rate of $44 \%$. The vast majority of respondents described themselves as glaucoma consultant ophthalmologists (71\%), followed by glaucoma specialist optometrists/consultant optometrists (14\%), specialist orthoptists $(6 \%)$, specialist registrars (3\%), other subspecialty consultant ophthalmologists (2\%), glaucoma fellows (2\%) advanced nurse practitioners (1\%) and academics (1\%).

The expert panel reviewed the scores and comments resulting from round 1, and developed a modified questionnaire consisting of 8 questions with a total of 21 statements for further scoring.

\section{Round 2}

The second, modified survey was circulated to UKEGS members for a 14 week period starting in October 2015, with 2 electronic reminders over this period. Once more, 86 members responded to the survey (glaucoma consultant ophthalmologists: $86 \%$, glaucoma specialist optometrists/consultant optometrists: $3.5 \%$, specialist orthoptists: $1 \%$, advanced nurse practitioners : $1 \%$, other subspecialty consultant ophthalmologists: $3.5 \%$, associate specialist: $1 \%$, specialist registrars: $1 \%$, glaucoma fellows: $1 \%$, academics: $1 \%$, not specified: $1 \%$ ). Table 1 illustrates the standards statements and scores following round 2 .

The expert panel reviewed and discussed the scores obtained in round 2 and developed a draft of the final standards document for consultation. 


\section{Stakeholder comments}

In March 2016, stakeholders were invited to comment upon the standards document drafted by the expert panel following round 2. After a 3 month consultation, the expert panel discussed stakeholder comments, addressed queries and made further edits and changes to the standards document. After a further month's consultation, the standards document was finalized and accepted by the Royal College of Ophthalmologists in December 2016 (Appendix 1).

\section{Discussion:}

This project has led to the development of UK national guidelines for the development and implementation of virtual clinics in glaucoma care in the secondary NHS setting.

The Delphi method is an accepted method for gaining consensus agreement between experts as it has a number of advantages. Firstly, having anonymity allows for all voices to be heard and avoids the potential for any one individual's opinions to dominate. Secondly, multiple iterations for feedback allow individuals to change their mind. Thirdly, assigning a 'score' to the level of agreement facilitates a statistical approach to measuring consensus. [18] This method has been used in ophthalmology to develop competencies for UK optometrists involved in glaucoma shared care, [17] to identify areas of consensus and disagreement in paediatric cataract management[19] and to define the minimum set of outcome measures required following cataract surgery. [20]

It may be noted that whilst the National Institute of Clinical and Healthcare Excellence CG85 guidelines recommend the use of Van Herick angle assessment and applanation tonometry in the assessment of the glaucoma patient, [21] these two techniques were removed from the final standards document following stakeholder consultation. Research suggests that with training, there is good agreement between observers in undertaking Van Herick angle measurement, [22, 23] but concerns were raised with respect to training and maintenance of standards, with the suggestion that anterior segment optical coherence tomography (AS-OCT) would allow for an objective recording of angle status. There is some evidence to suggest that the AS-OCT tends to overcall narrow angles, [24] but in the case of virtual clinics, it is probably better to 
have a false positive than false negative. Historically, applanation tonometry is the gold standard for measuring IOP, but there is plenty of evidence to show it is imprecise and inaccurate.[25-27] As such, it was not a recommended test in the final standards. Perhaps it is now the time to move on towards other validated techniques for IOP measurement, with full awareness of each of their relative merits and disadvantages. A leading hospital in Finland uses the rebound tonometer as standard, with the view that sustainable and safe eye care requires a 'good enough' approach to management, particularly with high-volume services. [28] It is important to make the distinction that the standards refer to care within the secondary care setting, and remains a hospital-led service, whereby overall responsibility for the patient lies with the secondary care team. Whilst aspects of the virtual clinic could be met in community, such as for the collection of IOP, visual field and optic disc imaging data, the reviewer will be based in the secondary care setting. This is a very different service delivery model to that of shared care, where the responsibility for the patient lies with the shared-care provider.

Virtual clinics appear to be increasing in popularity across the UK due to capacity pressures within NHS hospital eye service departments. They are seen as a model of service delivery that 'free up' consultant time to review more complex glaucoma patients. [12,29] The glaucoma virtual clinic model appears to be accepted by patients, particularly if they understand the status of their disease and current risk of progression to blindness. $[11,30]$ However, risk is not static, and glaucoma patients can quickly move from a 'stable' to 'unstable' status. Thus, an essential requirement of the virtual clinic model is to be able to pick up change in status. We anticipate that our standards will ensure that this minimum requirement is met, and hope that future research will evaluate whether delivery models that meet these standards are 'safe' for patients.

\section{Acknowledgments:}

The authors would like to that Tracey Fabre, Events and Marketing Co-ordinator for UKEGS for assisting us in administering the survey, and the NIHR Biomedical Research Centre for funding this project. Dr. Kotecha and Professor Foster receives a proportion of her funding from the Department of Health's National Institute for Health Research Biomedical Research Centre for Ophthalmology at Moorfields Eye Hospital NHS Foundation Trust and the UCL Institute of Ophthalmology. 


\section{References (I will format these for submission later)}

1. Pensions, D.o.W.a., Focus on older people, 2005, Department of Work and Pensions.

2. Tham, Y.C., et al., Global Prevalence of Glaucoma and Projections of Glaucoma Burden through 2040: A Systematic Review and Meta-Analysis. Ophthalmology, 2014.

3. Myint, J., et al., A national survey of diagnostic tests reported by UK community optometrists for the detection of chronic open angle glaucoma. Ophthalmic Physiol Opt, 2011. 31(4): p. 353-9.

4. Edgar, D.F., et al., Referral Behaviour Among Optometrists: Increase in the Number of Referrals from optometrists Following the Publication of the April 2009 NICE Guidelines for the Diagnosis and Management of COAG and OHT in England and Wales and its Implications. Optometry in Practice, 2010. 11(1): p. 33-38.

5. Ratnarajan, G., et al., The effect of changes in referral behaviour following NICE guideline publication on agreement of examination findings between professionals in an established glaucoma referral refinement pathway: the Health Innovation \&amp; Education Cluster (HIEC) Glaucoma Pathways project. Br J Ophthalmol, 2013. 97(2): p. 210-4.

6. Chalk, D. and M. Smith, Guidelines on glaucoma and the demand for services. British Journal of Healthcare Management, 2013. 19(10): p. 476-481.

7. Smith, R. Our ophthalmology service is "failing", please help! Professional Standards Committee, 15 August 2013.

8. Kotecha, A., et al., Improving care and increasing efficiency-challenges in the care of chronic eye diseases. Eye (Lond), 2014. 28(7): p. 779-83.

9. Trikha, S., et al., The Portsmouth-based glaucoma refinement scheme: a role for virtual clinics in the future? Eye (Lond), 2012. 26(10): p. 1288-94.

10. Kotecha, A., et al., Experiences with developing and implementing a virtual clinic for glaucoma care in an NHS setting. Clin Ophthalmol, 2015. 9: p. 1915-23.

11. Court, J.H. and M.W. Austin, Virtual glaucoma clinics: patient acceptance and quality of patient education compared to standard clinics. Clin Ophthalmol, 2015. 9: p. 745-9.

12. Clarke, J., et al., Virtual clinics in glaucoma care: face-to-face versus remote decision-making. Br J Ophthalmol, 2016.

13. Wright, H.R. and J.P. Diamond, Service innovation in glaucoma management: using a web-based electronic patient record to facilitate virtual specialist supervision of a shared care glaucoma programme. Br J Ophthalmol, 2015. 99(3): p. 313-7.

14. Tsaousis, K.T., et al., The concept of virtual clinics in monitoring patients with age-related macular degeneration. Acta Ophthalmol, 2016. 94(5): p. e353-5.

15. Bohmer, R.M.J., Leading Clinicians and Clinicians Leading. New England Journal of Medicine, 2013. 368(16): p. 1468-1470.

16. Hasson, F., S. Keeney, and H. McKenna, Research guidelines for the Delphi survey technique. J Adv Nurs, 2000. 32(4): p. 1008-15. 
17. Myint, J., et al., Development of a competency framework for optometrists with a specialist interest in glaucoma. Eye (Lond), 2010. 24(9): p. 1509-14.

18. Jones, J. and D. Hunter, Consensus methods for medical and health services research. BMJ, 1995. 311(7001): p. 376-80.

19. Serafino, M., et al., Use of the Delphi process in paediatric cataract management. Br J Ophthalmol, 2016. 100(5): p. 611-5.

20. Mahmud, I., et al., A Proposed Minimum Standard Set of Outcome Measures for Cataract Surgery. JAMA Ophthalmol, 2015. 133(11): p. 1247-52.

21. NICE. National Institute of Clinical Excellence (NICE) guidance CG85 Glaucoma: diagnosis and management of chronic open angle glaucoma and ocular hypertension. April 2009; Available from: http://guidance.nice.org.uk/CG85.

22. Campbell, P., et al., Repeatability and comparison of clinical techniques for anterior chamber angle assessment. Ophthalmic Physiol Opt, 2015. 35(2): p. 170-8.

23. Jindal, A., et al., Agreement among optometrists and ophthalmologists in estimating limbal anterior chamber depth using the van Herick method. Ophthalmic Physiol Opt, 2015. 35(2): p. 179-85.

24. Nolan, W.P., et al., Detection of primary angle closure using anterior segment optical coherence tomography in Asian eyes. Ophthalmology, 2007. 114(1): p. 33-9.

25. Whitacre, M.M. and R. Stein, Sources of error with use of Goldmann-type tonometers. Survey of Ophthalmology., 1993. 38(1): p. 1-30.

26. Ajtony, C., et al., Applanation tonometry: interobserver and prism agreement using the reusable Goldmann applanation prism and the Tonosafe disposable prism. Br J Ophthalmol, 2016. 100(6): p. 848-53.

27. Burr, J.M., et al., Surveillance for ocular hypertension: an evidence synthesis and economic evaluation. Health Technol Assess, 2012. 16(29): p. 1-271, iiiiv.

28. Tuulonen, A., et al., Right services to right patients at right time in right setting in Tays Eye Centre. Acta Ophthalmol, 2016. 94(7): p. 730-735.

29. Committee, R.P.S., New to Followup ratios in ophthalmology outpatient services, 2011.

30. Kotecha, A., et al., Qualitative investigation of patients' experience of a glaucoma virtual clinic in a specialist ophthalmic hospital in London, UK. BMJ Open, 2015. 5(12): p. e009463. 